\title{
Discursos de Greta Thunberg: Análisis de contenido y su impacto en YouTube
}

\section{Greta Thunberg Speeches: Content analysis and its impact on YouTube}

Lucía Espejel Gómez*

Universidad Anáhuac México

Av. Universidad Anáhuac núm. 46, Col. Lomas Anáhuac, Huixquilucan, Estado de México, C.P. 52786

Jorge Alberto Hidalgo Toledo**

Universidad Anáhuac México

Av. Universidad Anáhuac núm. 46, Col. Lomas Anáhuac,

Huixquilucan, Estado de México, C.P. 52786

\section{Editor: Rogelio del Prado Flores}

Fecha de recepción: 30 de abril de 2020

Fecha de aceptación: 9 de mayo de 2020 lucia.espejelgo@anahuac.mx https://orcid.org/0000-0003-0430-3677

jhidalgo@anahuac.mx https://orcid.org/0000-0002-6204-9534

https://doi.org/10.36105/stx.2020n5.03

\section{RESUMEN}

La presente investigación tiene por objeto analizar la correlación positiva espacio-tiempo entre los catorce discursos de Greta Thunberg y los eventos más importantes de cambio climático en el periodo septiembre 2018 a septiembre 20I9. El evento disparador de

* Doctoranda en Investigación de la Comunicación por la Universidad Anáhuac México, Maestra en Ética Aplicada por la Universidad de Lovaina, Maestra en Administración de Empresas por la Universidad de Tulane. Ha trabajado en empresas de clase mundial como: Celanese Mexicana, Ford Motor Company, General Electric (GE), BASF, Alberto Culver, Embajada Británica en áreas de Investigación y Desarrollo, Planeación Estratégica, Mercadotecnia y Gestión de Proyectos.

** Doctor en Comunicación Aplicada por la Universidad Anáhuac México; Miembro del Sistema Nacional de Investigadores Nivel I. Vicepresidente de la Asociación Mexicana de Investigadores de la Comunicación (AMIC) para el periodo 2017-2019; Vicepresidente Universitario de la Academia Mexicana de la Comunicación para el periodo 2015-20I7 y 2017-2020; Presidente del Consejo Nacional para la Enseñanza e Investigación de las Ciencias de la Comunicación (CONEICC) para el periodo 20I2-20I5; Secretario de la Asociación Católica de Comunicación, SIGNIS México, para el periodo 20I4-20I6. Cuenta con veinte años de experiencia profesional en áreas de comunicación; tres libros publicados y más de treinta capítulos en libros nacionales e internacionales. 
estos discursos fue la presentación del informe del Panel Intergubernamental de Cambio Climático (PICC) en octubre de 2018 , donde presentó que el nuevo objetivo de incremento de temperatura para este siglo es de $\mathrm{I} .5{ }^{\circ} \mathrm{C}$ y no de $2{ }^{\circ} \mathrm{C}$ como se había acordado en la reunión de París en 20I5. El enfoque de los discursos es principalmente científico, presentando argumentos simples y en línea con la información del último reporte del PICC; en segundo lugar, el enfoque es social y en tercer lugar, es político, seguido del enfoque económico. Además, se hace un análisis de contenido de sentimientos de varios discursos. Se utilizó la metodología de análisis de contenido de Krippendorff y un software especializado para la sistematización de documentos y su metaanálisis. Se seleccionaron los tres discursos de Greta con más visitas en YouTube. Los principales hallazgos fueron: existe una correlación positiva entre el tono más negativo de los discursos con I) el número de visitantes a los diversos canales de YouTube donde se transmite el discurso; 2 ) el incentivo que tienen los espectadores a dar un "me gusta" o "no me gusta"; 3 ) el aumento de la proporción de "no me gusta”. En resumen, cuanto más apocalíptico el discurso, más visitas tiene, aunque le gusta menos a los visitantes de YouTube.

Palabras clave: cambio climático, líder de opinión, influencers, análisis de sentimientos, redes sociales.

\section{ABSTRACT}

This paper analyzes the positive space-time correlation between the fourteen speeches of Greta Thunberg and climate change milestones during September 2018-September 2019. The trigger event was the launch of the Intergovernmental Panel on Climate Change (IPCC) report in October 20I8, where it presented that the new objective of temperature increase for this century is $1.5^{\circ} \mathrm{C}$ and not $2{ }^{\circ} \mathrm{C}$ as agreed at the Paris meeting in 2015. The approach of the speeches is mainly scientific, presenting simple arguments and aligned with the latest IPCC report, secondly, the approach is social and thirdly, political, followed by the economic approach. An emotional content analysis is performed based on Krippendorff methodology by utilizing a specialized software. Three Greta speeches were selected based on the highest number of YouTube visits. The main findings are: there is a positive correlation between negative tone of the speeches with I) the number of visitors to the various YouTube channels where the speech is transmitted; 2) the participation of users with "like" or "dislike"; 3) increases the proportion of "dislikes". Hence, the more apocalyptic the speech, the more visitors it has, and the more "dislikes" in YouTube.

Keywords: climate change, opinion leader, influencers, sentiment analysis, social media. 


\section{INTRODUCCIÓN}

$\mathrm{L}$ a comunicación de cambio climático tradicionalmente se ha conocido como divulgación científica. El problema de la divulgación científica está en el enfoque informacional con el que históricamente viene siendo encarado y desarrollado. En la actualidad ha surgido una nueva disciplina que es comunicación de la ciencia, todavía incipiente y entendida como un proceso interactivo y normativo de compartir y de producir conocimiento en colaboración entre los diversos actores involucrados. En lugar de la recurrente discusión de la divulgación científica se propone pensar en una comunicación de la ciencia (Malcher y Cunha, 20I3).

El objetivo principal de la investigación es encontrar una relación espacio tiempo entre los hitos de cambio climático y los discursos de Greta Thunberg en el periodo 20I8-20I9 y el análisis de contenido de tres de sus discursos para comprender la estrategia de comunicación de los organismos internacionales acerca del cambio climático hacia los jóvenes en el mundoy entender el rol de Greta Thunberg comolíder de opinión o influencer social. Se decidió acotar el estudio a análisis de contenido y no un análisis completo del discurso, para encontrar si existía una correlación entre contenidos presentados por el PICC y la actuación de la activista como propagadora de esos contenidos. Además, el interés por conocer si con el análisis de sentimientos se puede relacionar al número de visitas del video y el impacto que tiene en la audiencia.

Los objetivos específicos de esta investigación fueron los siguientes:

I. Medir la claridad de la comunicación e identificar el tipo de enfoque (económico, social, político, científico).

2. Analizar el contenido y evaluar el tono de los discursos (positivo-neutral-negativo).

3. Medir el impacto y recepción que desencadenó cada discurso en YouTube.

4. Encontrar la relación que existe entre el contenido de cada discurso y los eventos importantes mundiales relacionados con el cambio climático.

Las hipótesis fueron las siguientes:

- Hipótesis I $\left(\mathrm{H}_{\mathrm{I}}\right)$. Existe una correlación en espacio y tiempo entre eventos importantes de cambio climático acordes al Panel Intergubernamental de Cambio Climático (PICC) y los discursos de Greta Thunberg.

- Hipótesis $2\left(\mathrm{H}_{2}\right)$. El tono de los discursos de Greta Thunberg tiene una correlación con el número de visitas y evaluaciones de los visitantes al sitio de YouTube. Cuanto más negativo es el tono, mayor número de visitas tiene. 
- Hipótesis $3\left(\mathrm{H}_{3}\right)$. Cuanto más negativo es el tono de los discursos, aumenta la proporción de "no me gusta" con respecto a "me gusta" en la evaluación de los visitantes al sitio de YouTube.

\section{ESTADO DEL ARTE}

\section{La comunicación científica y el cambio climático}

Actualmente se habla de comunicación científica en lugar de divulgación científica, con lo que se enfatiza una relación más horizontal con la intención de aproximar, compartir y estimular el debate; a diferencia de la verticalidad del experto transfiriendo conocimientos con enfoque informacional.

Las investigaciones recientes sugieren un cambio en el enfoque en cuanto al compromiso y el aprendizaje en los problemas de comunicación. Así, la conversación ha pasado de informar y educar a las masas ignorantes a involucrar a las personas en el tema del cambio climático (Wibeck, 20I4), (Stocknes, 20I4).

La comunicación de la ciencia es todavía incipiente y propositivamente entendida como un proceso interactivo y normativo de compartir y de producir conocimiento en colaboración entre los diversos actores involucrados en una investigación: investigadores y públicos directa o indirectamente involucrados en la organización (Malcher y Cunha, 2013).

Los autores Malcher y Cunha hacen una reflexión acerca del concepto de divulgación científica, tejiendo una discusión teórica de la construcción, aún incipiente, de una noción de la comunicación de la ciencia como proceso de coparticipación y producción colaborativa de conocimiento científico que se diferencia, por lo tanto, de una visión funcionalista en el que la comunicación, en el contexto científico, se asocia apenas a actividades de divulgación de resultados de investigación (Malcher y Cunha, 20I3).

En la literatura, el cambio climático se describe como un fenómeno complejo, generalizado e incierto que las personas encuentran difícil de entender, conceptualizar y relacionar (Lorenzoni, 2006). Es un tema caracterizado por la incertidumbre, la controversia, el escepticismo y el compromiso limitado de las personas. La falta de compromiso y acción a menudo se atribuyen al hecho de que el cambio climático se percibe como distante en el tiempo y en el espacio, lo que permite concluir de que el cambio climático no representa una amenaza personal visible comparado con otros problemas más urgentes (Lorenzoni, Nicholson-Cole, y Whitmarsh, 2007).

Massarani y Moreira (2005) presentan las tres líneas más comunes de lo que denominan comunicación científica: la primera sería formada por discursos científicos primarios, 
producidos por científicos para otros científicos; la segunda se refiere a discursos científicos didácticos que, generalmente, componen manuales de enseñanza; y la tercera línea sería la de los discursos científicos divulgativos, de la llamada divulgación científica, propiamente dicha, dirigida a públicos no iniciados.

Los discursos de Greta Thunberg caen en la tercera categoría.

\section{MARCO TEÓRICO Y CONTEXTUAL}

El cambio climático es el mayor desafío de nuestro tiempo y nos encontramos en un momento decisivo. Si no se toman medidas drásticas desde hoy, será más difícil y costoso adaptarse a estos efectos en el futuro (ONU, 2Or8).

El cambio climático es complejo porque es un problema mundial, multifactorial y transversal por lo que el proyecto de solución debe ser uno de los más ambiciosos que se hayan planteado en la historia de la humanidad. El problema es de difícil solución porque los países más ricos del mundo, los que generan la mayor cantidad de gases de efecto invernadero, son los que iniciaron este problema y aumenta con el crecimiento económico de los países asiáticos, principalmente China e India, con lo que la amenaza crece como una bola de nieve, y más grave aún, puede volverse irreversible.

Como todos los problemas multifactoriales, las soluciones son complejas y con participación de todos los grupos de interés. Mario Molina afirma que los científicos pueden plantear los problemas que afectarán al medio ambiente con base en la evidencia disponible, pero su solución no es responsabilidad de los científicos, es de toda la sociedad (Molina, 20I8).

El Panel Intergubernamental de Cambio Climático (PICC), organismo creado por la Organización de Naciones Unidas (ONU), define cambio climático como una importante variación estadística en el estado medio del clima o en su variabilidad, que persiste durante un período prolongado (normalmente decenios o incluso más). El cambio climático se puede deber a procesos naturales internos o a cambios del forzamiento externo, o bien a cambios persistentes antropogénicos en la composición de la atmósfera (IPCC, 20I8).

E18 de octubre de 20I8, el Grupo Intergubernamental de Expertos sobre el Cambio Climático de la ONU pidió contener el aumento de la temperatura promedio global a I.5 grados por encima de los niveles preindustriales y no a 2 grados como se estableció en el Acuerdo de París. Cumplir con el objetivo de I.5 grados mantendría la subida del nivel global del mar en o.I metros por debajo del objetivo de 2 grados para 2100, según el reporte. Esto podría reducir las inundaciones y dar tiempo para adaptarse a la gente que vive en las costas, islas y 
deltas fluviales de todo el mundo. También se reduciría la pérdida de especies y la extinción y el impacto en los ecosistemas (Forbes, 20I8).

La activista climática sueca Greta Thunberg inició la Huelga Escolar por el Clima el 20 de agosto de 2018 en su ciudad natal Mynttorget, Estocolmo. Pronunció su primer discurso público el 8 de septiembre de 2018 en Estocolmo en el marco de La marcha de los Pueblos por el Clima (Kuhne, 2019). En este primer discurso público señaló que su motivación para comenzar el Huelga Escolar por el Clima fue un artículo científico que se publicó en junio de 2017 y que declaró que solo había tres años para alcanzar el objetivo del acuerdo de París de 2015 para mantener el calentamiento global por debajo de dos centígrados por encima de los niveles preindustriales de 1850 (Figueres, 2017).

Greta ha ofrecido I 4 discursos en dos años, en el periodo de septiembre de 20I8-septiembre de 2019 y ha sido un fenómeno mediático y una influencer en los jóvenes preocupados por el cambio climático.

\section{METODOLOGÍA}

Del total de discursos ofrecidos por Greta Thunberg desde octubre de 2018 a septiembre de 20I9, se seleccionaron tres discursos con base en mayor número de visitantes en YouTube.

I. Se relacionaron las fechas de estos discursos con eventos importantes del Panel Intergubernamental de Cambio Climático (PICC).

2. Se obtuvo la transcripción de los discursos. Se analizó el contenido de éstos, con el conteo de palabras con más menciones por medio de un software.

3. Se realizó el análisis de sentimientos, mediante la identificación de tono de discurso (positivo, neutral, negativo).

4. Se identificaron los contenidos de los discursos de acuerdo al enfoque (económico, social, político, científico).

5. Se siguió el método de los ocho aspectos de un proyecto de un análisis de contenido (Krippendorf, 1990).

- Aplicación del marco de referencia conceptual al análisis de contenido.

- Búsqueda de los datos adecuados.

- Búsqueda del conocimiento contextual.

- Desarrollo de planes para la determinación de las unidades y el muestreo. 
- Desarrollo de las instrucciones de codificación.

- Búsqueda de procedimientos justificados según el contexto.

- Establecimiento de las normas de calidad.

\section{ANÁLISIS DE SENTIMIENTOS}

$\mathrm{El}$ análisis de sentimientos es el proceso de determinar el tono emocional de una serie de palabras en menciones en un texto o en un discurso. Los humanos son bastante intuitivos al interpretar el tono de un discurso de un escrito. Desde el punto de vista de la minería de textos, el análisis de sentimientos es una tarea de clasificación masiva de documentos de manera automática, en función de la connotación positiva o negativa del lenguaje ocupado en el documento. Estos tratamientos generalmente "se basan en relaciones estadísticas y de asociación, no en análisis lingüístico". Pang realizó uno de los primeros trabajos en clasificar la polaridad de los sentimientos (positivo, negativo y neutro) (Pang, Lee, y Vaithyanathan, 2002).

\section{RESULTADOS Y ANÁLISIS}

Greta Thunberg ha ofrecidoI4 discursos en el periodo 20I8-2019. Los primeros diez se llevaron a cabo en Europa, después tres en Estados Unidos y el último en Canadá.

TABLA 1. DISCURSOS DE GRETA THUNBERG 2018-2019

\begin{tabular}{|c|c|c|c|}
\hline NúM. & FECHA & SEDE & CIUDAD, PAÍS \\
\hline 1 & 20 de agosto de 2018 & $\begin{array}{l}\text { People Climate March } \\
\text { Marcha de los pueblos por el clima }\end{array}$ & $\begin{array}{l}\text { Mynttorget, } \\
\text { Estocolmo }\end{array}$ \\
\hline 2 & 31 de octubre de 2018 & $\begin{array}{l}\text { Extinction Rebellion } \\
\text { Rebelión de Extinción }\end{array}$ & $\begin{array}{l}\text { Londres, } \\
\text { Reino Unido }\end{array}$ \\
\hline 3 & 24 de noviembre de 2018 & TEDxStockholm & Estocolmo, Suecia \\
\hline 4 & $\begin{array}{l}4 \text { de diciembre de } 2018 \\
20 \text { de diciembre de } 2018\end{array}$ & $\begin{array}{l}\text { COP24 summit } \\
\text { (Cumbre Cambio Climático COP24) }\end{array}$ & Katowice, Polonia \\
\hline 5 & 23 de enero de 2019 & Davos & Davos, Suiza \\
\hline
\end{tabular}




\begin{tabular}{|c|c|c|c|}
\hline NÚM. & FECHA & SEDE & CIUDAD, PAÍS \\
\hline 6 & 21 de febrero de 2019 & $\begin{array}{l}\text { European Economic and Social Committee } \\
\text { Comité Económico y Social Europeo }\end{array}$ & Bruselas, Bélgica \\
\hline 7 & 29-31 de marzo de 2019 & $\begin{array}{l}\text { Berlin } \\
\text { (Puerta de Branderburgo) }\end{array}$ & Berlín, Alemania \\
\hline 8 & 23 de abril de 2019 & $\begin{array}{l}\text { EU leaders } \\
\text { (Parlamento Europeo) }\end{array}$ & Estrasburgo, Francia \\
\hline 9 & 29 de mayo de 2019 & $\begin{array}{l}\text { Austrian World Summit R20 } \\
\text { Cumbre mundial austriaca R20 }\end{array}$ & Viena, Austria \\
\hline 10 & 21 de julio de 2019 & $\begin{array}{l}\text { Prix Liberté Normandy: } 2019 \text { Freedom Prize } \\
\text { Premio Libertad Normandía } 2019\end{array}$ & Normandía, Francia \\
\hline 11 & 18 de septiembre de 2019 & $\begin{array}{l}\text { U.S. Congress on climate change } \\
\text { Cambio climático en el Congreso de EUA }\end{array}$ & Congreso de EUA \\
\hline 12 & 20 de septiembre de 2019 & $\begin{array}{l}\text { New York City, USA: } 2019 \text { Global Climate } \\
\text { Strike } \\
\text { Huelga climática global } 2019\end{array}$ & Nueva York, EUA \\
\hline 13 & 23 de septiembre de 2019 & $\begin{array}{l}\text { United Nations: Climate Action Summit } 2019 \\
\text { onU: Cumbre de Cambio Climático } 2019\end{array}$ & $\begin{array}{l}\text { Naciones Unidas, } \\
\text { Nueva York, EUA }\end{array}$ \\
\hline 14 & 27 de septiembre de 2019 & $\begin{array}{l}\text { Montreal, Canada: } 2019 \text { Global Climate Strike } \\
\text { Huelga climática global } 2019\end{array}$ & Montreal, Canadá \\
\hline
\end{tabular}

FUENTE: ELABORACIÓN PROPIA.

Existe correlación entre espacio y tiempo en los discursos que ofreció Greta Thunberg. El evento más importante sucedió en octubre de 2018 cuando el PICC publicó su nuevo reporte y anunció el nuevo límite de incremento de temperatura en este siglo, disminuyendo a I. $5^{\circ} \mathrm{C}$ y no el de $2{ }^{\circ} \mathrm{C}$ que se ratificó en el acuerdo de París en el 2015. A partir de este hito, se desencadenaron los discursos de Greta Thunberg a la par de los eventos más importantes de cambio climático. En dos ocasiones coincidió el discurso de Greta con el evento: I) La Cumbre mundial austriaca R2O y 2) La Cumbre de Cambio Climático en EUA. En la Figura I se muestra la línea de tiempo de los eventos y los discursos. 
FIGURA 1. DISCURSOS DE GRETA THUNBERG CON RELACIÓN A HITOS DE CAMBIO CLIMÁTICO 2018-2019

\section{Discursos Greta - Hitos de cambio climático 2018-2019}

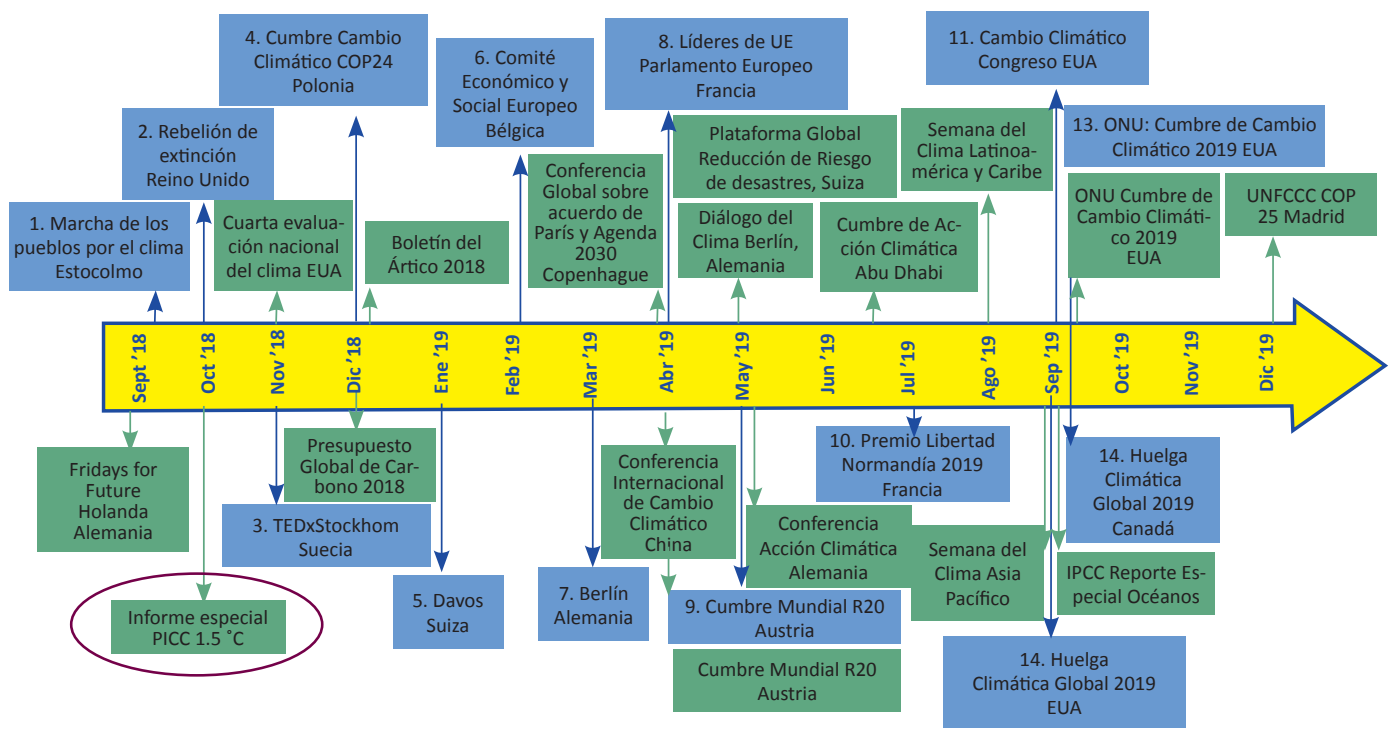

FUENTE: ELABORACIÓN PROPIA BASADA EN (ROADMAP TO CLIMATE ACTION SUMMIT 2019, 2019).

En la Tabla 2 se presentan los resultados del impacto de los discursos de Greta Thunberg representados por el número de visitas a los canales de YouTube que transmitieron los discursos y el número de opiniones de "me gusta" o "no me gusta". El discurso que más visitas tuvo fue el de la Cumbre de Cambio Climático 2019 de la ONU en Nueva York, el 23 de septiembre de 2019, con un número de visitas de 7,732,821 y 61\% de visitantes que les gustó el discurso y $39 \%$ que no les gustó. En segundo lugar se encuentra la cumbre de Cambio Climático $\mathrm{COP}_{24}$ en Katowice, Polonia, el I4 de diciembre de 20I8. El discurso tuvo un total de I, 542,433 visitas, $88 \%$ de personas que les gustó y I $2 \%$ que no les gustó. En tercer lugar está el discurso a los líderes del Parlamento Europeo ofrecido el 23 de abril de 2019 en Estrasburgo, Francia. El total de visitas en los canales de YouTube fue de I,089,729 y de los visitantes que mostraron su interés, a $84 \%$ le gustó y I $6 \%$ no le gustó el discurso. 
TABLA 2. DISCURSOS SELECCIONADOS Y MÉTRICAS DE IMPACTO EN CANALES DE YOUTUBE

\begin{tabular}{|c|c|c|c|c|c|c|c|}
\hline NÚM. & FECHA & REFERENCIA & $\begin{array}{c}\text { CANAL } \\
\text { YOUTUBE }\end{array}$ & SUSCRIPTORES & VISITAS & "ME GUSTA" & $\begin{array}{l}\text { "NO ME } \\
\text { GUSTA" }\end{array}$ \\
\hline 4 & $\begin{array}{l}\text { Dic 15, } \\
2018\end{array}$ & $\begin{array}{c}\text { (Connect4Climate } \\
\text { 2018) }\end{array}$ & $\begin{array}{l}\text { Connect } 4 \\
\text { Climate }\end{array}$ & 11,200 & $1,526,321$ & $\begin{array}{r}20,791 \\
(88 \%)\end{array}$ & $\begin{array}{l}2,807 \\
(12 \%)\end{array}$ \\
\hline 4 & $\begin{array}{l}\text { Dic 14, } \\
2018\end{array}$ & $\begin{array}{l}\text { (FridaysForFuture, } \\
\text { 2018) }\end{array}$ & $\begin{array}{l}\text { FridaysFor } \\
\text { Future }\end{array}$ & 1,910 & 12,039 & $\begin{array}{c}136 \\
(94 \%)\end{array}$ & $\begin{array}{c}9 \\
(6 \%)\end{array}$ \\
\hline 4 & $\begin{array}{l}\text { Dic 14, } \\
2018\end{array}$ & (CykelPendlare, 2018) & $\begin{array}{l}\text { Cykel } \\
\text { Pendlare }\end{array}$ & 124 & 4,073 & $\begin{array}{c}81 \\
(95 \%)\end{array}$ & $\begin{array}{c}4 \\
(5 \%)\end{array}$ \\
\hline 4 & $\begin{array}{l}\text { Dic 14, } \\
2018\end{array}$ & Total Discurso Núm. 4 & & 13,234 & $1,542,433$ & $\begin{array}{c}21,008 \\
(88 \%)\end{array}$ & $\begin{array}{l}2,820 \\
(12 \%)\end{array}$ \\
\hline 8 & $\begin{array}{l}\text { Abril 16, } \\
2019\end{array}$ & $\begin{array}{c}\text { (GuardianNews, Greta } \\
\text { Thunberg's emotional } \\
\text { speech to EU leaders, } \\
\text { 2019) }\end{array}$ & $\begin{array}{l}\text { Guardian } \\
\text { News }\end{array}$ & 612,000 & 770,148 & $\begin{array}{c}22,528 \\
(84 \%)\end{array}$ & $\begin{array}{l}4,265 \\
(16 \%)\end{array}$ \\
\hline 8 & $\begin{array}{l}\text { Abril 16, } \\
2019\end{array}$ & (APF, 2019) & $\begin{array}{l}\text { APF news } \\
\text { agency }\end{array}$ & 488,000 & 15,566 & $\begin{array}{c}152 \\
(66 \%)\end{array}$ & $\begin{array}{c}78 \\
(34 \%)\end{array}$ \\
\hline 8 & $\begin{array}{c}\text { Abril 23, } \\
2019\end{array}$ & $\begin{array}{l}\text { (FridaysForFuture, } \\
\text { Greta Thunberg } \\
\text { full speech at the } \\
\text { EU Parliament in } \\
\text { Strasbourg, 2019) }\end{array}$ & $\begin{array}{l}\text { FridaysFor } \\
\text { Future }\end{array}$ & 1,910 & 19,336 & $\begin{array}{c}212 \\
(88 \%)\end{array}$ & $\begin{array}{c}29 \\
(12 \%)\end{array}$ \\
\hline 8 & $\begin{array}{l}\text { Abril 23, } \\
2019\end{array}$ & (GuardianNews, 2019) & $\begin{array}{l}\text { Guardian } \\
\text { News }\end{array}$ & 612,000 & 194,986 & $\begin{array}{l}1,942 \\
(85 \%)\end{array}$ & $\begin{array}{c}331 \\
(15 \%)\end{array}$ \\
\hline 8 & $\begin{array}{l}\text { Abril 24, } \\
2019\end{array}$ & (WWFUK, 2019) & WWF UK & 15,800 & 89,693 & $\begin{array}{l}1,309 \\
(81 \%)\end{array}$ & $\begin{array}{c}299 \\
(11 \%)\end{array}$ \\
\hline 8 & & Total Discurso Núm. 8 & & $1,729,710$ & $1,089,729$ & $\begin{array}{c}26,143 \\
(84 \%)\end{array}$ & $\begin{array}{l}5,002 \\
(16 \%)\end{array}$ \\
\hline 13 & $\begin{array}{l}\text { Sep 23, } \\
2019\end{array}$ & $\begin{array}{l}\text { (GuardianNews, Greta } \\
\text { Thunberg to world } \\
\text { leaders: 'How dare } \\
\text { you? You have stolen } \\
\text { my dreams and my } \\
\text { childhood', 2019) }\end{array}$ & $\begin{array}{l}\text { Guardian } \\
\text { News }\end{array}$ & 611,000 & $3,901,732$ & $\begin{array}{l}51,438 \\
(64 \%)\end{array}$ & $\begin{array}{c}29,299 \\
(36 \%)\end{array}$ \\
\hline
\end{tabular}


56 LUCíA ESPEJEL GÓMEZ - JORGE ALBERTO HIDALGO TOLEDO

\begin{tabular}{|c|c|c|c|c|c|c|c|}
\hline NÚM. & FECHA & REFERENCIA & $\begin{array}{c}\text { CANAL } \\
\text { YOUTUBE }\end{array}$ & SUSCRIPTORES & VISITAS & "ME GUSTA" & $\begin{array}{l}\text { "NO ME } \\
\text { GUSTA" }\end{array}$ \\
\hline 13 & $\begin{array}{l}\text { Sep 23, } \\
2019\end{array}$ & (PBSNewsHour, 2019) & $\begin{array}{l}\text { PBS News } \\
\text { Hour }\end{array}$ & $1,460,000$ & $2,424,247$ & $\begin{array}{c}42,346 \\
(68 \%)\end{array}$ & $\begin{array}{l}20,261 \\
(32 \%)\end{array}$ \\
\hline 13 & $\begin{array}{c}\text { Sep } \\
23 \\
2019\end{array}$ & $\begin{array}{l}\text { (UnitedNations, } \\
\text { 2019) }\end{array}$ & $\begin{array}{l}\text { United } \\
\text { Nations }\end{array}$ & 597,000 & 751,086 & $\begin{array}{l}7,080 \\
(53 \%)\end{array}$ & $\begin{array}{l}6,207 \\
(47 \%)\end{array}$ \\
\hline 13 & $\begin{array}{c}\text { Sep } \\
23 \\
2019\end{array}$ & $\begin{array}{c}\text { (TheTelegraph, } \\
\text { 2019) }\end{array}$ & $\begin{array}{l}\text { The } \\
\text { Telegraph }\end{array}$ & 727,000 & 655,756 & $\begin{array}{l}5,944 \\
(31 \%)\end{array}$ & $\begin{array}{r}13,237 \\
(69 \%)\end{array}$ \\
\hline 13 & $\begin{array}{c}\text { Sep } \\
23 \\
2019\end{array}$ & $\begin{array}{l}\text { Total Discurso } \\
\text { Núm. } 13\end{array}$ & & $3,395,000$ & $7,732,821$ & $\begin{array}{c}106,808 \\
(61 \%)\end{array}$ & $\begin{array}{c}69,004 \\
(39 \%)\end{array}$ \\
\hline
\end{tabular}

FUENTE: ELABORACIÓN PROPIA.

En la Tabla 3 se presentan los resultados de análisis de sentimientos (negativo-neutral-positivo) y la proporción de visitantes que evaluaron con "me gusta"/ "no me gusta".

TABLA 3. CLASIFICACIÓN DE ANÁLISIS DE SENTIMIENTOS DE LOS DISCURSOS

\begin{tabular}{|c|c|c|c|c|c|c|c|}
\hline NÚM. & DISCURSO & NEGATIVO & NEUTRAL & POSITIVO & TOTAL & $\begin{array}{l}\text { POSITIVO/ } \\
\text { NEGATIVO }\end{array}$ & $\begin{array}{c}\text { YOUTUBE } \\
\text { ME GUSTA/ } \\
\text { NO ME GUSTA }\end{array}$ \\
\hline 4 & $\begin{array}{l}\text { Cumbre Cambio Climático } \\
\text { COP24 }\end{array}$ & $37 \%$ & $43 \%$ & $20 \%$ & $100 \%$ & 0.54 & 7.4 \\
\hline 8 & Líderes del Parlamento Europeo & $22 \%$ & $66 \%$ & $12 \%$ & $100 \%$ & 0.55 & 5.2 \\
\hline 13 & $\begin{array}{l}\text { ONU: Cumbre de Cambio } \\
\text { Climático } 2019\end{array}$ & $37 \%$ & $50 \%$ & $13 \%$ & $100 \%$ & 0.37 & 1.5 \\
\hline
\end{tabular}

FUENTE: ELABORACIÓN PROPIA. 
TABLA 4. EJEMPLO DE LA CLASIFICACIÓN DE ACUERDO CON LOS SENTIMIENTOS

\begin{tabular}{|c|l|l|l|}
\hline NÚM. & \multicolumn{1}{|c|}{ DISCURSO } & \multicolumn{1}{|c|}{ MENSAJE } & \multicolumn{1}{|c|}{ SENTIMIENTO } \\
\hline 4 & $\begin{array}{l}\text { Cumbre Cambio Climático } \\
\text { COP24 }\end{array}$ & $\begin{array}{l}\text { "He aprendido que nunca eres demasiado pequeño } \\
\text { para marcar la diferencia" }\end{array}$ & Positivo \\
\hline 8 & $\begin{array}{l}\text { Líderes del Parlamento } \\
\text { Europeo }\end{array}$ & $\begin{array}{l}\text { "Nuestro futuro se ha vendido para que un } \\
\text { puñado de personas puedan ganar cantidades } \\
\text { inimaginables de dinero" }\end{array}$ & Negativo \\
\hline 8 & $\begin{array}{l}\text { Líderes del Parlamento } \\
\text { Europeo }\end{array}$ & $\begin{array}{l}\text { "Pero nosotros solo repetimos el mensaje de la } \\
\text { ciencia sobre el clima" }\end{array}$ & Neutro \\
\hline
\end{tabular}

FUENTE: ELABORACIÓN PROPIA.

En la Tabla 5 y la Figura 2 se identifica el contenido del discurso número 4. El enfoque es simple, más social; seguido de científico, después político y al final económico. Los discursos número 8 y I3 son principalmente con enfoque científico; después social, en tercer lugar, político y el último económico. Todos los discursos presentan la misma información que contiene el último reporte del PICC.

TABLA 5. CLASIFICACIÓN DE ENFOQUE DE LOS DISCURSOS

\begin{tabular}{|c|c|c|c|}
\hline NÚM. & DISCURSO & ENFOQUE & PORCENTAJE \\
\hline 4 & Cumbre Cambio Climático COP24 & $\begin{array}{l}\text { Científico } \\
\text { Social } \\
\text { Político } \\
\text { Económico }\end{array}$ & $\begin{array}{l}27 \% \\
42 \% \\
18 \% \\
13 \%\end{array}$ \\
\hline 8 & Líderes del Parlamento Europeo & $\begin{array}{l}\text { Científico } \\
\text { Social } \\
\text { Político } \\
\text { Económico }\end{array}$ & $\begin{array}{c}61 \% \\
20 \% \\
10 \% \\
9 \%\end{array}$ \\
\hline 13 & $\begin{array}{l}\text { ONU: Cumbre de Cambio Climático } \\
2019\end{array}$ & $\begin{array}{l}\text { Científico } \\
\text { Social } \\
\text { Político } \\
\text { Económico }\end{array}$ & $\begin{array}{l}75 \% \\
14 \% \\
7 \% \\
5 \%\end{array}$ \\
\hline
\end{tabular}

FUENTE: ELABORACIÓN PROPIA. 
58 LUCía ESPEJEL GÓMEZ - JORGE ALBERTO HIDALGO TOLEDO

FIGURA 2. ENFOQUE DE LOS DISCURSOS (CIENTÍFICO-SOCIAL-POLÍTICO-ECONÓMICO)

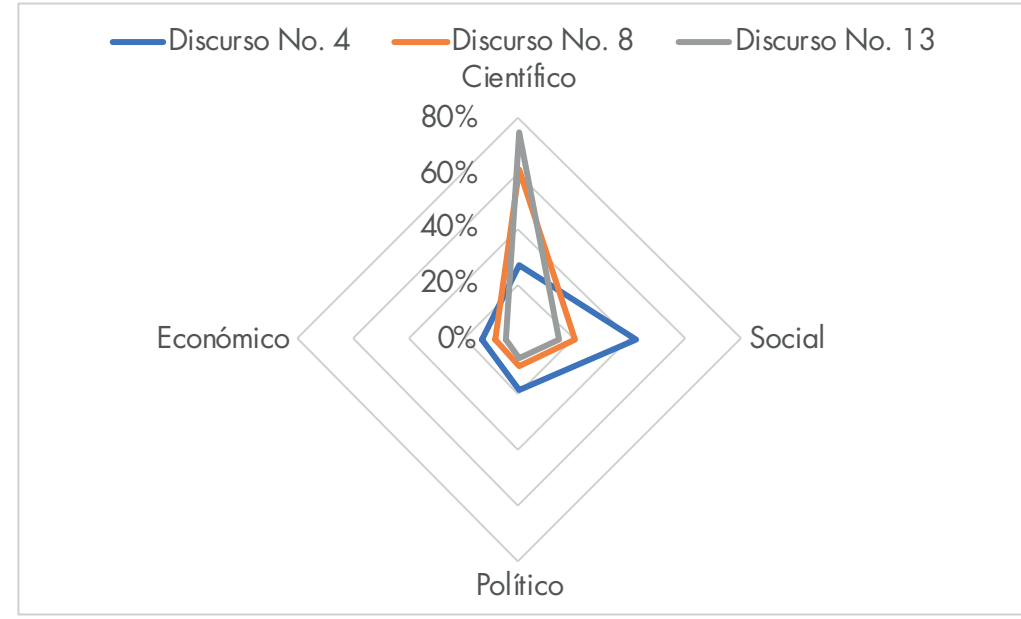

En la Figura 3 se muestra el gráfico de tono de discurso (positivo-neutro-negativo) y el número de visitas con el respectivo porcentaje de "me gusta", "no me gusta".

FIGURA 3. MAPA PERCEPTUAL ANÁLISIS DE SENTIMIENTOS VS. IMPACTO EN YOUTUBE VS "ME GUSTA" / "NO ME GUSTA"

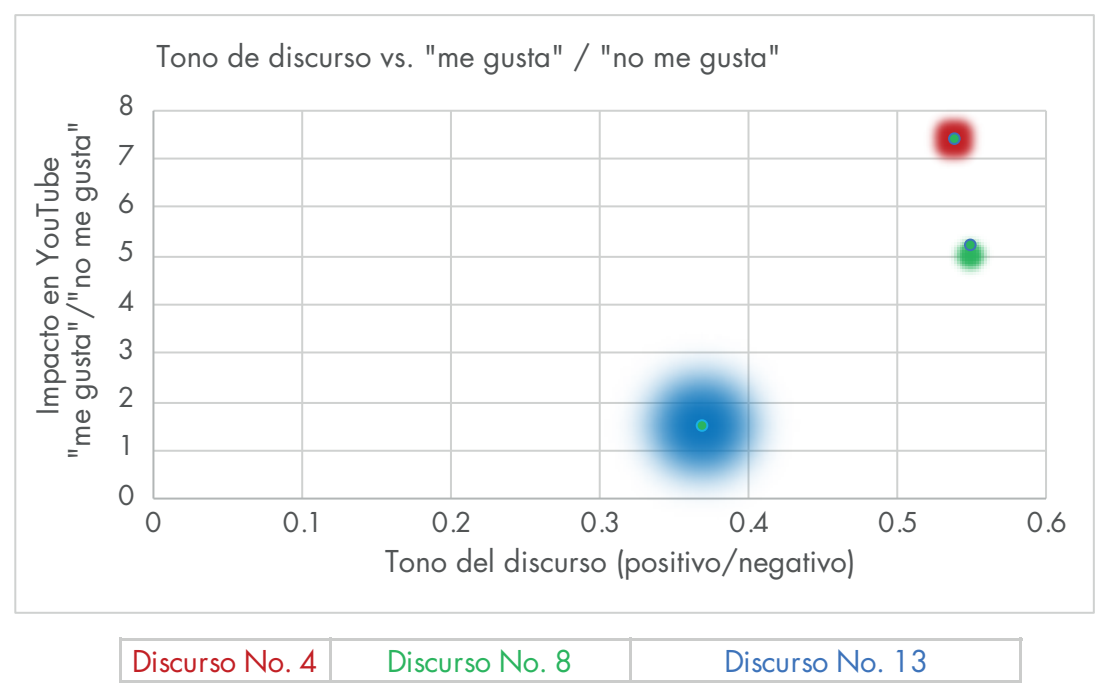

Discurso 4: Cumbre Cambio Climático COP24 (rojo); Discurso 8: Líderes del Parlamento Europeo (verde); Discurso I3 ONU: Cumbre de Cambio Climático 2019 (azul). FUENTE: ELABORACIÓN PROPIA. 


\section{CONCLUSIONES}

El uso de una metodología mixta en este estudio permite identificar los siguiente: I) el rol y el timing de la activista para comunicar y reforzar conceptos de carácter económico, político, social y científico recientemente publicados por el PICC; y 2) el análisis de sentimientos en cuanto al tono del discurso y su impacto en número de visitas y en el que la audiencia esté dispuesta a expresar su opinión a través de "me gusta" o "no me gusta".

Existe una correlación positiva en espacio y tiempo entre los discursos de Greta Thunberg y eventos importantes de cambio climático de organismos internacionales como la ONU y la emisión de reportes del PICC. Los discursos de Greta son concisos, simples y poderosos. El tono es de indignación y denuncia a los actuales líderes del mundo que por la ambición de ganar más dinero están destruyendo el futuro de los jóvenes. En el análisis de contenido se observa que tiene un enfoque científico, con argumentos poderosos del PICC, como la reducción de emisiones de bióxido de carbono, uno de los gases de efecto invernadero, que más efecto tienen en la crisis climática. En segundo lugar, está el de impacto social y en tercer lugar el enfoque político y por último el económico.

En cuanto al análisis de sentimientos, los resultados muestran que cuanto más negativo es el tono de los discursos, mayor número de visitas tiene en YouTube y promueve la participación de los visitantes para votar con "me gusta" o "no me gusta". Cuanto más apocalíptico es el discurso, mayor número de visitas tiene en YouTube, menos les gusta a los visitantes, pero participan más. Posiblemente este es el objetivo: crear más conciencia entre los seguidores, aunque no les guste el discurso.

En poco más de un año, Greta Thunberg se ha convertido en un fenómeno social; un actor político joven en la cultura contemporánea, y una influencer social mundial no sólo de los jóvenes de su generación (generación Z que nacieron entre 1996 y el 2020), sino también de los millennials (generación y que nacieron entre 1981 y 1995). Las acciones discursivas de Greta Thunberg tienen las siguientes características: I) se alinean con la información que va actualizando el PICC y sirven para amplificar su discurso a audiencias jóvenes; y 2) actúan como catalizador para crear interés entre la audiencia e impulsar el deseo entre la población de participar en acciones de mitigación ante el cambio climático y 3) convertirse a estos jóvenes en promotores de la transición energética hacia las energías renovables dejando atrás el uso de combustibles fósiles aplanando el terreno a los lobbistas para influir ante la Administración Pública para promover decisiones favorables a los intereses de magnates; empresarios; economistas; brokers; políticos; abogados; y empresas de tecnología, informática, bienes raíces, aseguradoras, y todas la que integran la cadena de valor de esta nueva industria energética verde. Greta Thunberg es una pieza clave del poder blando de esta revolución energética. 
Esta investigación solo analiza tres de los catorce discursos de Greta. Sería interesante analizar todos los discursos para obtener más datos estadísticos y correlaciones.

Este estudio tiene una gran área de oportunidad para indagar la reacción de los usuarios en otras redes sociales como Twitter o Facebook, donde los participantes tienen la oportunidad de expresar su opinión con respecto a los discursos de manera más explícita que el solo "me gusta" o "no me gusta". Para futuras investigaciones se sugiere hacer una minería de datos de análisis de sentimientos con otros medios sociales.

\section{REFERENCIAS}

APF. (I6 de abril de 2019). Greta Thunberg gives emotional speech at EU Parliament. Recuperado de https://www.Youtube.com/watch?v=sZIRNUp4DgU

Connect4Climate. (I5 de diciembre de 20I8). Greta Thunberg full speech at UN Climate Change $\mathrm{COP}_{24}$ Conference. Recuperado de https://www.Youtube.com/watch?v=VFkQSGyeCWg

CykelPendlare. (I4 de diciembre de 20I8). Greta Thunberg COP 24 , I2th December, 20I8. Recuperado de https://www.Youtube.com/watch?v=Kp2pQnPtceo

Dave. (ro de enero de 2019). Top ro Climate Change Milestones in 2018-\#climate. Skeptical Science. Promoting Science and Critical Tbinking. Recuperado de https://www.skeptical-science.com/ science/top-Io-climate-change-milestones-in-20I8-climate/

Fernández, F. (2002). El análisis de contenido como ayuda metodológica. Redalyc, 35-53.

Figueres, C. (2017). Three years to safeguard our climate. Nature, 546, 593-595.

Forbes. ( 8 de octubre de 2018). ONU urge a no permitir un calentamiento global mayor a I.5 grados. Forbes México. Recuperado de https:/www.forbes.com.mx/onu-urge-a-no-permitir-uncalentamiento-global-mayor-a-I-5-grados/

FridaysForFuture. (I4 de diciembre de 20I8). Greta Thunberg addressed the $\mathrm{COP}_{24}$ plenary session December I2th. Recuperado de https://www.Youtube.com/watch?v=Ziznxp8b65 E

FridaysForFuture. (23 de abril de 2019). Greta Thunberg full speech at the EU Parliament in Strasbourg. Recuperado de https://www.Youtube.com/watch?v=cJAcuQEVxTY

GuardianNews. (23 de abril de 2019). Greta Thunberg tells MPs: “Our future was sold”. Recuperado de https://www.Youtube.com/watch?v=FwYo6xE24Es

GuardianNews. (23 de septiembre de 2019). Greta Thunberg to world leaders: How dare you? You have stolen my dreams and my childhood'. Recuperado de https:/www.Youtube.com/ watch? $=$ TMrtLsQbaok

GuardianNews. (I6 de abril de 20I9). Greta Thunberg's emotional speech to EU leaders. Recuperado de https://www.Youtube.com/watch?v=FWsMg-_zrKo

Hulme, M. (2009). Why we disagree about climate change: Understanding controversy, inaction and opportunity. Cambridge, UK. Cambridge University Press, 392.

IPCC. (29 de noviembre de 20I8). Recuperado de IPCC: https://www.ipcc.ch/pdf/glossary/tar-ipccterms-sp.pdf 
Krippendorf, K. (1990). Metodología de análisis de contenido. Teoría y práctica. Buenos Aires: Paidós Comunicación.

Kuhne, R. (2019). Climate Change: The Science Bebind Greta Tbunberg and Fridays For Future. https://doi.org/IO.I3I40/RG.2.2.21256.78087

Lorenzoni, I. (2006). Public Views on Climate Change: European and USA Perspectives. Climate Change, 77 .

Lorenzoni,I., Nicholson-Cole, S.,y Whitmarsh,L. (2007). Barriers perceived to engaging with climate change among the UK public and their policy implications. Global Environmental Cbange, 445-449.

Malcher, M., y Cunha, S. (2013). Construyendo una noción de la comunicación de la Ciencia. Cbiespal. Ensayos, 74-8I.

Molina, M. (2018). Centro Mario Molina para Estudios Estratégicos sobre Energía y Medio Ambiente. Recuperado de https://centromariomolina.org/taller-de-actualizacion-sobre-cambio-climatico/

Organización de las Naciones Unidas (ONU). (29 de noviembre de 20I8). Recuperado de http://www.un.org/es/sections/issues-depth/climate-change/index.html

Pang, B. P., Lee, L., y Vaithyanathan, S. (2002). Thumbs up? Sentiment Classification using Machine Learning Techniques. Proceedings of the 2002 Conference on Empirical Methods in Natural Language Processing, 79-86. Association for Computational Linguistics. https://doi.org/I0.3115/III8693.1118704

PBSNewsHour. (23 de septiembre de 20I9). WATCH: Greta Thunberg's full speech to world leaders at UN Climate Action Summit. Recuperado de https://www.Youtube.com/watch?v=KAJsdgTPJpU

Ramírez, R. T. (2015). La mary el ancla. La educación ambiental en la administración pública en México. Ciudad de México: La Zonámbula.

Rebeil, C. M. (20I4). Para una comprensión de la teoría práctica de la comunicación. Redalyc.

(2019). Roadmap to Climate Action Summit 20Ig. NY: UN. Recuperado de https://www.un.org/en/ climatechange/assets/pdf/Climate_Action_Summit_20I9_Road\%20Map_May_20I9.pdf

Steffen, W., Rockström, J., Richardson, K., Lenton, T., Folke, C., Liverman, D., Barnosky, A. D. (20I8). Trajectories of the Earth System in the Anthropocene. (W. C. Clark, Ed.) II5(33), 82528259. https://doi.org/https://doi.org/IO.IO73/pnas.I8IoI4III5

Stocknes, P. (20I4). Rethinking climate communications and the "psychological climate paradox". Energy Research and Social Science, I6 $1-170$.

TheTelegraph. (23 de septiembre de 2019). Emotional Greta Thunberg attacks world leaders: "How dare you?”. Recuperado de https://www.Youtube.com/watch?v=xVlRompciyE

UnitedNations. (23 de septiembre de 20I9). Greta Thunberg (Young Climate Activist) at the Climate Action Summit 20I9-Official Video. Recuperado de https://www.Youtube.com/watch?$\mathrm{v}=\mathrm{u}_{9} \mathrm{KxE}_{4} \mathrm{Kv}_{9} \mathrm{~A} 8$

Wibeck, V. (20I4). Enhancing learning, communication and public engagement about climate change-some lessons from recent literature. Environmental Education Research, 387-4II.

WWFUK. (24 de abril de 20I9). Greta Thunberg full speech to UK Parliament | Climate strikes. Recuperado de https://www.Youtube.com/watch?v=rYNM4rsnNFM 